\title{
Análise de métodos de estimativa de evapotranspiração na otimização de sistemas de drenagem
}

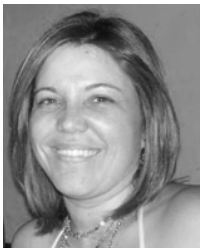

\author{
Késia O. da Silva ${ }^{1}$, Jarbas H. de Miranda ${ }^{2}$, Sergio N. Duarte ${ }^{3}$ \& Marcos V. Folegatti ${ }^{3}$ \\ 1 Instituição Moura Lacerda, Ribeirão Preto, SP, Av. Dr. Oscar de Moura Lacerda n.1520, CEP:14.076-510, Ribeirão \\ Preto, SP, Tel/Fax (16) 627-0276, E-mail: kosilva@uol.com.br (Foto) \\ 2 Departamento de Ciências Exatas. ESALQ/USP, Fone: (19) 3429-4283, Ramal: 210. E-mail: jhmirand@esalq.usp.br \\ ${ }^{3}$ Departamento de Engenharia Rural. ESALQ/USP, Fone: (19) 3429-4217. E-mail: snduarte@esalq.usp.br; \\ mvfolega@esalq.usp.br
}

Protocolo 112 - 6/4/2003 - Aprovado em 29/10/2004

\begin{abstract}
Resumo: Inúmeros são os métodos para se estimar a evapotranspiração potencial (ETP) em que os mais simplificados apresentam limitações quanto à precisão dos resultados obtidos e os mais complexos apontam a dificuldade de se dispor de todos os dados necessários ao cálculo. No presente trabalho, compararam-se os métodos de Thornthwaite e Camargo com o de PenmanMonteith, tomado como padrão, com o objetivo de se verificar qual a influência da utilização de cada método na estimativa da ETP diária sobre o dimensionamento econômico de um sistema de drenagem, utilizando-se o modelo SISDRENA. Pelos resultados obtidos concluiu-se que as diferenças entre os métodos não afetaram significativamente a obtenção do espaçamento econômico entre drenos.
\end{abstract}

Palavras-chave: modelagem, dimensionamento econômico, espaçamento entre drenos

\section{Analysis of methods to estimate evapotranspiration for optimization of drainage systems}

\begin{abstract}
There are various methods to estimate the potential evapotranspiration (ETP). The simplified methods present limitations in relation to precision of the obtained results, while the more complex methods present the difficulty of availability of necessary data for calculation. In the present study the Thornthwaite and Camargo methods were compared with the PenmanMonteith method to verify the influence in obtaining daily ETP for the design of a drainage system, using the SISDRENA model. It is concluded that the differences among the methods did not affect significantly the calculation of economic drain spacing.
\end{abstract}

Key words: modeling, economic design, drain spacing

\section{INTRODUÇÃO}

A água é o componente mais abundante nas plantas, representando de 80 a $90 \%$ do peso fresco na maioria das espécies herbáceas, além de ser responsável pelo mantimento da turgescência, ou pressão nas células, atuando também como solvente na maior parte dos solutos presentes nas mesmas consumindo, ainda, uma porção considerável da energia solar incidente pela evaporação nas folhas (transpiração), evitando que esquentem excessivamente. Considerando-se que, em geral, é difícil separar os processos de evaporação e transpiração, mutuamente dependentes, estes são reunidos e tratados como se fossem um único processo, chamado evapotranspiração.
Segundo Pereira et al. (1997), a evapotranspiração é controlada pela disponibilidade de energia, pela demanda atmosférica e pelo suprimento de água do solo às plantas. Existem diversos métodos para a determinação da evapotranspiração potencial, cada um com suas limitações. Cabe ao usuário, então, decidir qual deles utilizar, de acordo com a sua conveniência.

Segundo Santiago (2001), devido à complexidade e ao alto custo dos dispositivos de medidas da ETo, inúmeros são os métodos de estimativa, bem como, farta é a disponibilidade de textos sobre o assunto entre os quais destacamse Gangopadhyaya et al. (1966), Penman et al. (1967), Jensen (1973), Jensen et al. (1990), Doorenbos \& Pruitt (1977), Berlato \& Molion (1981), Brutsaert (1982), Villa Nova \& Reichardt (1989), Camargo \& Sentelhas (1997), Pereira et al. (1997), Medeiros (1998) e Allen et al. (1998). 
Camargo \& Sentelhas (1997) avaliaram o desempenho de vinte métodos de estimativa da evapotranspiração potencial (ETP), em três localidades do Estado de São Paulo, mediante dados obtidos em lisímetros conduzidos entre 1954 e 1960. Os dados medidos foram correlacionados com os estimados por meio de regressão linear. As melhores estimativas de evapotranspiração, para as condições do clima subtropical úmido do interior do Estado de São Paulo, foram obtidas pelos métodos de Camargo, Thornthwaite, Thornthwaite com índice de calor "T" e Priestley \& Taylor. Os métodos de PenmanMonteith, Penman, Hargreaves modificado, Makking e Blaney $\&$ Criddle modificado, tiveram bom desempenho. Os demais métodos mostraram desempenho bem inferior.

No presente trabalho foram comparados os métodos de Thornthwaite e Camargo com o de Penman-Monteith, objetivando-se verificar qual a influência da utilização de cada método de estimativa da evapotranspiração potencial na obtenção do espaçamento entre drenos, mais econômico.

\section{MATERIAL E MÉTODOS}

Realizaram-se simulações utilizando-se dados climáticos diários da região de Piracicaba, SP (Lat.22 $42^{\prime}$ 'S, Long.47²3 ' W e altitude de $546 \mathrm{~m}$ ) constituindo uma série histórica de 16 anos (1979 a 1994). A evapotranspiração potencial foi determinada pelos métodos de Thornthwaite, Camargo e Penman-Monteith.

O método de Thornthwaite está representado pelas Eqs. 1 e 2 :

$$
\begin{gathered}
\mathrm{ETP}=16\left(10 \frac{\mathrm{Tn}}{\mathrm{I}}\right)^{\mathrm{a}} \quad 0 \leq \mathrm{Tn}<26,5{ }^{\circ} \mathrm{C} \\
\mathrm{ETP}=-415,85+32,24 \mathrm{Tn}-0,43 \mathrm{Tn}^{2} \quad \mathrm{Tn} \geq 26,5^{\circ} \mathrm{C}
\end{gathered}
$$

em que:

ETP - evapotranspiração potencial padrão, $\mathrm{mm} \mathrm{d}^{-1}$

Tn - temperatura média do mês, ${ }^{\circ} \mathrm{C}$

I - índice que expressa o nível de calor disponível na região

Segundo Pereira et al. (1997), o valor de I depende do ritmo anual da temperatura (preferencialmente com valores normais), sendo o efeito térmico de cada mês calculado pela Eq. 3 .

$$
\mathrm{I}=\sum_{\mathrm{n}=1}^{12}(0,2 \mathrm{Tn})^{1,514}
$$

O expoente "a" é função de I, e também é um índice térmico regional podendo ser calculado pela Eq. 4:

$$
\mathrm{a}=6,75 \cdot 10^{-7} \mathrm{I}^{3}-7,71 \cdot 10^{-5} \mathrm{I}^{2}+1,7912 \cdot 10^{-2} \mathrm{I}+0,49239
$$

O método de Camargo está representado pela Eq. 5:

$$
\mathrm{ETP}=0,01 \mathrm{Q}_{\mathrm{o}} \mathrm{T} \mathrm{ND}
$$

em que:

$\mathrm{Q}_{0}$ - irradiância solar global extraterrestre, $\mathrm{mm} \mathrm{d}^{-1}$

$\mathrm{T}$ - temperatura média do ar, ${ }^{\circ} \mathrm{C}$

ND - número de dias do período considerado

O método de Penman-Monteith está representado pela Eq. 6:

$$
\mathrm{ETP}=\frac{0,408 \mathrm{~s}(\mathrm{Rn}-\mathrm{G})+\frac{\gamma 900 \mathrm{U}_{2}\left(\mathrm{e}_{\mathrm{s}}-\mathrm{e}_{\mathrm{a}}\right)}{\mathrm{T}+273}}{\mathrm{~s}+\gamma\left(1+0,34 \mathrm{U}_{2}\right)}
$$

em que:

$\mathrm{Rn}$ - saldo de radiação diária, $\mathrm{MJ} \mathrm{m}^{-2} \mathrm{~d}^{-1}$

$\mathrm{G}$ - fluxo de calor no solo, $\mathrm{MJ} \mathrm{m}^{-2} \mathrm{~d}^{-1}$

$\gamma$ - fator psicrométrico $\left(0,063 \mathrm{kPa}^{\circ} \mathrm{C}^{-1}\right)$

$\mathrm{U}_{2}$ - velocidade do vento a $2 \mathrm{~m}$ de altura, $\mathrm{m} \mathrm{s}^{-1}$

$\mathrm{e}_{\mathrm{s}}$ - pressão de saturação de vapor, $\mathrm{kPa}$

$\mathrm{e}_{\mathrm{a}}$ - pressão parcial de vapor, $\mathrm{kPa}$

$\mathrm{s}$ - declividade da curva de saturação do vapor d'água, $\mathrm{kPa}{ }^{\circ} \mathrm{C}^{-1}$

As simulações envolveram três tipos de solo de perfil homogêneo e $5 \mathrm{~m}$ de profundidade, apresentando condutividade hidráulica saturada de 1,0 $\mathrm{m} \mathrm{d}^{-1}$ (Solo 1); $0,5 \mathrm{~m} \mathrm{~d}^{-1}$ (Solo 2) e $0,1 \mathrm{~m} \mathrm{~d}^{-1}$ (Solo 3), cujas curvas de retenção são apresentadas por Miranda (1997). O modelo SISDRENA utiliza uma modificação do método do número da curva para estimar o escoamento superficial.

Os valores dos coeficientes $(\mathrm{CN})$ aplicados foram $64,85 \mathrm{e}$ 90 para os solos 1, 2 e 3, respectivamente. Foram simulados espaçamentos entre drenos abertos de 10 a $100 \mathrm{~m}$, com intervalos de $10 \mathrm{~m}$.

A Figura 1 apresenta os parâmetros geométricos dos drenos e seus respectivos valores.

Considerou-se que uma cultura de cana-de-açúcar com ciclo de um ano seria plantada em outubro e colhida em setembro, com três estádios de desenvolvimento definidos por Scardua (1985): estabelecimento vegetativo (125 dias), formação da produção (120 dias) e maturação (120 dias).

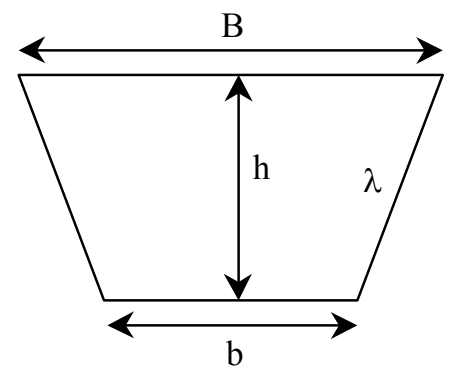

$\mathrm{B}-\mathrm{B}=(\mathrm{b}+(2 \cdot \mathrm{h} \cdot \lambda))+1,0(\mathrm{~m})$

$\mathrm{h}-1,4 \mathrm{~m}$

$\lambda$ - 1,25:1 (solo 1), 1,0:1 (solo 2) e 0,75:1 (solo 3)

Figura 1. Representação esquemática dos parâmetros geométricos dos drenos abertos 
Os valores diários de evapotranspiração obtidos pelos diferentes métodos foram aplicados para contabilizar os efeitos da deficiência hídrica nos respectivos estádios da planta, utilizando-se a função de produção proposta por Scardua (1985), cuja expressão apresenta coeficientes referentes a cada estádio de desenvolvimento da cultura (Eq. 7).

$$
\mathrm{Y}=0,0620+0,6610 \mathrm{X}_{1}+0,2762 \mathrm{X}_{2}-0,0306 \mathrm{X}_{3}
$$

O valor de quebra na produção, obtido pela Eq. 7, permitiu que se estimasse o valor da produtividade relativa ao déficit utilizando-se a Eq. 8 e o déficit de evapotranspiração dos $1^{\circ}, 2^{\circ}$ e $3^{\circ}$ estádios decimal, pode ser obtido pela Eq. 9.

$$
\begin{gathered}
\mathrm{YRD}=(1-\mathrm{Y}) \cdot 100 \\
\mathrm{X}_{1}, \mathrm{X}_{2}, \mathrm{X}_{3}=\left(1-\frac{\sum \mathrm{ETR}}{\sum \mathrm{ETP}}\right)
\end{gathered}
$$

em que:

Y - quebra de produção da cultura da cana-de-açúcar devido ao déficit de umidade, decimal

YRD - produtividade relativa ao déficit, $\%$

EETR - somatório da evapotranspiração real anual, de acordo com cada estádio de desenvolvimento da cultura da cana, $\mathrm{mm} \mathrm{d}^{-1}$

¿ETP - somatório da evapotranspiração potencial anual, de acordo com cada estádio de desenvolvimento da cultura da cana, $\mathrm{mm} \mathrm{d}^{-1}$

$$
\mathrm{X}_{1}, \mathrm{X}_{2}, \mathrm{X}_{3} \text { - parâmetros da função de produção }
$$

Os valores de $\Sigma$ ETR foram fornecidos pelo modelo SISDRENA, realizando-se um balanço hídrico na zona radicular da cultura. A produtividade relativa ao excesso foi calculada por uma relação experimental obtida por Carter (1985) (Eq. 10).

$$
\mathrm{YRW}=100-0,02771 . \mathrm{SEW}_{30}
$$

em que:

YRW - produtividade relativa ao excesso, \%

Esta equação possui, como parâmetro básico, o índice $\mathrm{SEW}_{30}$, que contabiliza o efeito do excesso de água na produtividade da cultura implantada sob condições em que o lençol freático atinja a sua zona radicular. De posse dos valores das produtividades relativas ao déficit e ao excesso torna-se possível calcular a produtividade relativa total, Eq. 11.

$$
\mathrm{YT}=\frac{\mathrm{YRD} \cdot \mathrm{YRW}}{100}
$$

em que:

YT - produtividade relativa total, $\%$
Com os valores obtidos de YT (\%) para cada ano, calculouse o valor médio (YTm) e o valor esperado com $80 \%$ de probabilidade de sucesso $\left(\mathrm{YT}_{80}\right)$. De posse dos valores de $\mathrm{YT}_{80}$ para cada espaçamento entre drenos, procedeu-se à análise econômica baseada no cálculo do índice valor presente.

\section{RESULTADOS E DISCUSSÃO}

Os métodos de Thornthwaite e Camargo foram comparados com o método de Penman-Monteith, pelo fato deste último ser bastante utilizado atualmente devido à sua maior confiabilidade. Assim, obtiveram-se as dispersões dos valores de ETP pelos respectivos métodos (Figura 2).

Observou-se que o coeficiente de determinação para a dispersão dos dados do método de Camargo em relação ao método de Penman-Monteith, foi um pouco superior ao do método de Thornthwaite. Esse resultado deve-se, possivelmente, ao fato dos dois primeiros métodos considerarem o efeito da radiação diretamente, enquanto o método de Thornthwaite baseia-se apenas na temperatura do ar.

Verificou-se tendência dos métodos de Thornthwaite e Camargo fornecerem estimativas de evapotranspiração potencial inferiores àquelas obtidas pelo método de PenmanMonteith.

Para auxiliar na discussão dos resultados obtidos na análise econômica, procurou-se avaliar os efeitos da aplicação dos diferentes métodos nos índices YRD, YRW e YTm.
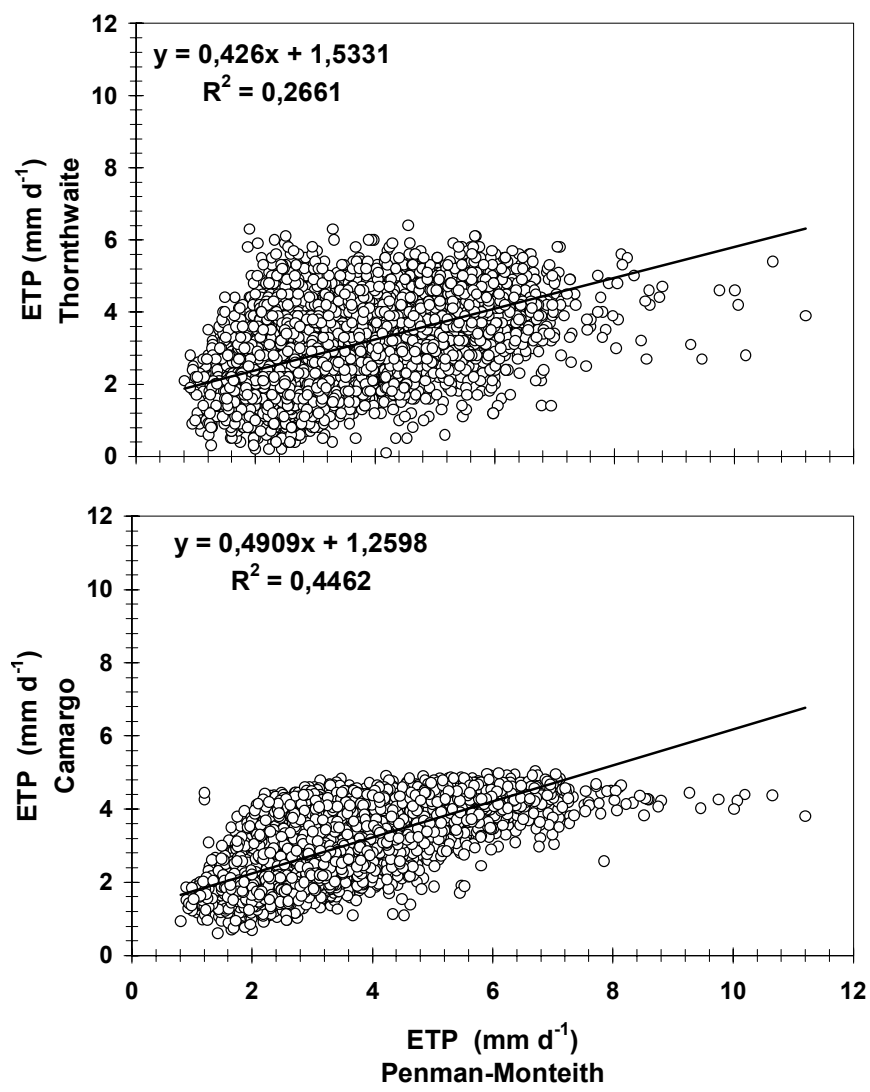

Figura 2. Valores diários de evapotranspiração potencial para o período de 1979-1994, obtidos pelos métodos de Thornthwaite e Camargo, tomando-se como base o método de Penman-Monteith, para Piracicaba, SP 


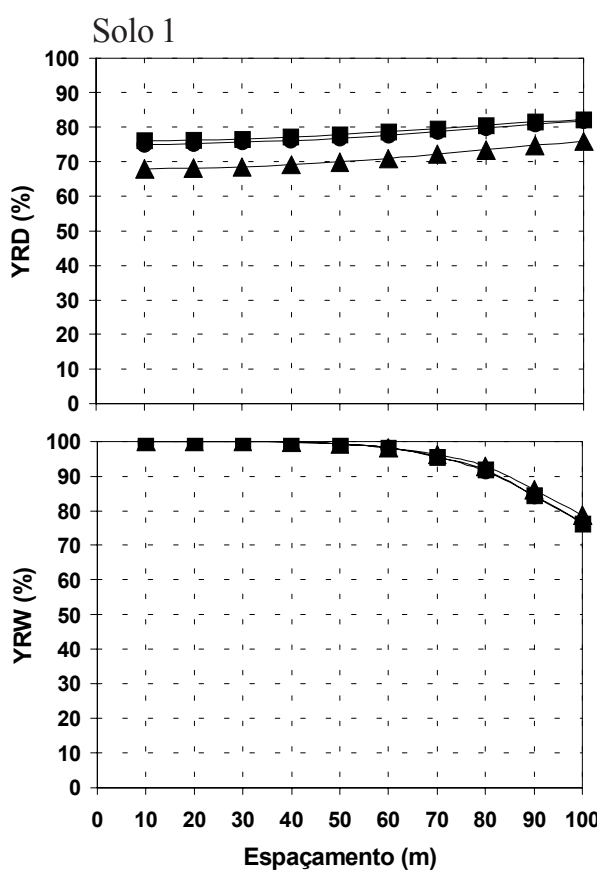

Solo 2

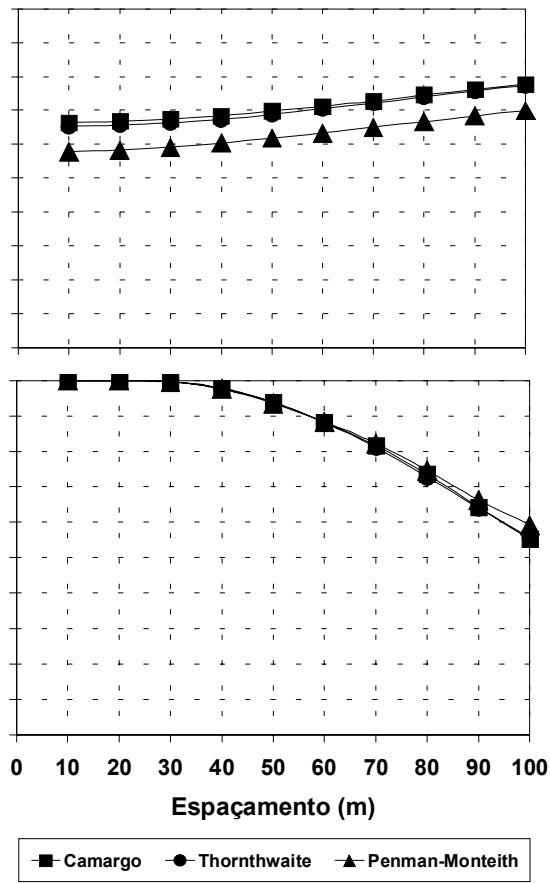

Solo 3
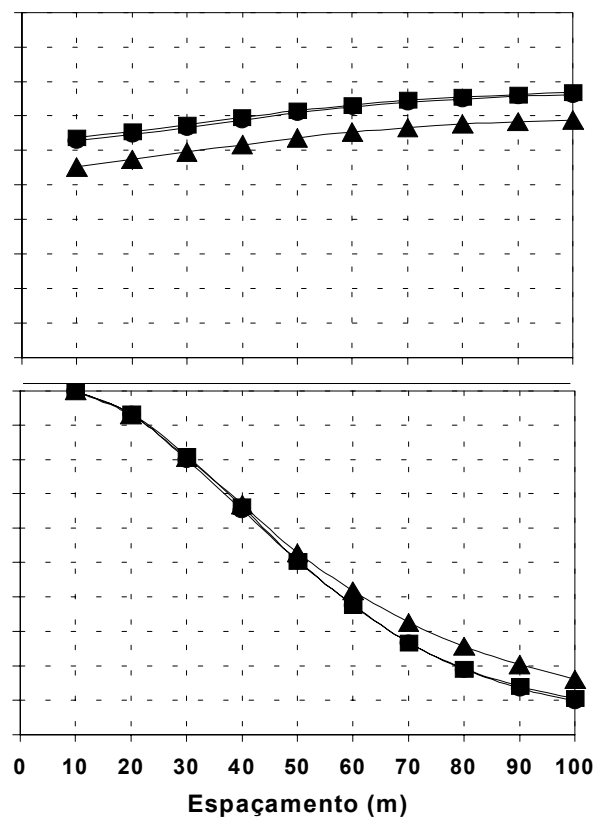

Figura 3. Representação esquemática dos valores médios de YRD e YRW para os solos 1, 2, 3 e respectivos espaçamentos

Constata-se, pela Figura 3, que o uso do método de Thornthwaite e Camargo gerou maiores valores de produtividade relativa da cultura devido à falta de água (YRD), principalmente para espaçamentos entre drenos menores. Por outro lado, o índice YRW foi pouco influenciado pelos diferentes métodos de estimativa da ETP. As maiores diferenças foram obtidas para os maiores espaçamentos entre drenos, condição em que o efeito do excesso de água é mais acentuado. Verificase que os métodos de Thornthwaite e Camargo resultaram em maiores valores de YTm, cujo resultado deve-se ao fato desses métodos terem gerado uma superestimativa de YRD maior que a subestimativa de YRW. Procedendo-se às análises econômicas baseadas nos valores de $\mathrm{YT}_{80}$, obtiveram-se os resultados apresentados na Tabela 1: para o solo 1, os métodos de Thornthwaite e Camargo não causaram diferenças sensíveis em relação ao método de Penman-Monteith, pois apresentaram espaçamentos mais econômicos de 60 e 50 m, respectivamente, enquanto o método de Penman-Monteith forneceu o valor de $60 \mathrm{~m}$ e para o solo 2, o método de Camargo apresentou o mesmo espaçamento econômico entre drenos, em relação ao método de Penman-Monteith, ou seja $50 \mathrm{~m}$; enfim, para o solo 3 não ocorreu diferença no espaçamento econômico determinado pelos métodos de Thornthwaite e Camargo, em relação ao método de Penman-Monteith (Tabela 1).

Vê-se, também, que, devido ao fato de subestimativa de ETP, os métodos de Thornthwaite e Camargo superestimaram ligeiramente as produtividades relativas com $80 \%$ de probabilidade de sucesso para os espaçamentos mais econômicos.

A análise econômica para obtenção dos espaçamentos entre drenos mais viáveis pode ser verificada mediante a observação da Tabela 1, na qual se nota que as diferenças entre os métodos de estimativa de evapotranspiração foram amenizadas, ou seja, os espaçamentos mais econômicos são aproximadamente semelhantes para os três métodos.
Tabela 1. Espaçamentos entre drenos mais econômicos, valores de produtividade relativa com $80 \%$ de probabilidade e valores presentes para os três tipos de solo simulados na localidade de Piracicaba, SP, utilizando-se dados diários de ETP obtidos pelos métodos de Penman-Monteith, Thornthwaite e Camargo

\begin{tabular}{cccc} 
Solo & $\begin{array}{c}\text { Espaçamentos } \\
(\mathrm{m})\end{array}$ & $\begin{array}{c}\mathrm{YT}_{80} \\
(\%)\end{array}$ & $\begin{array}{c}\text { Valor Presente } \\
\left(\mathrm{R} \$ \text { ha }^{-1}\right)\end{array}$ \\
\hline \multicolumn{3}{c}{ Penman-Monteith } \\
1 & 60 & 60,91 & $4.476,92$ \\
2 & 50 & 52,76 & $2.426,25$ \\
3 & 20 & 48,59 & 383,59 \\
\hline \multicolumn{3}{c}{ Thornthwaite } \\
1 & 60 & 69,91 & $6.726,30$ \\
2 & 30 & 63,58 & $4.248,61$ \\
3 & 20 & 55,21 & $1.869,97$ \\
\hline \multicolumn{3}{c}{ Camargo } \\
1 & 50 & 72,33 & $7.033,85$ \\
2 & 50 & 60,25 & $4.293,48$ \\
3 & 20 & 56,91 & $2.251,67$ \\
\hline
\end{tabular}

\section{CONCLUSÕES}

1. Os valores de evapotranspiração obtidos pelos métodos de Thornthwaite e Camargo subestimaram, de maneira geral, aqueles obtidos pelo método de Penman-Monteith. Esses valores refletiram uma tendência nítida de subestimar o efeito do estresse por falta d'água, acarretando produtividades superiores aos obtidos com o método de Penman-Monteith.

2. Ao realizar a análise econômica com vistas a obter os espaçamentos entre drenos mais viáveis economicamente, verificou-se que essas diferenças foram amenizadas e que, 
portanto, os valores de espaçamentos entre drenos foram aproximadamente semelhantes para os três métodos, evidenciando que não há influência do método selecionado na simulação.

3. Existe viabilidade na aplicação de métodos menos complexos, tais como Thornthwaite e Camargo, para a determinação dos valores diários de evapotranspiração potencial, visando ao dimensionamento econômico de sistemas de drenagem.

\section{LITERATURA CITADA}

Allen, RG.; Pereira, L.S.; Raes, D.; Smith, M. Crop evapotranspiration: guidelines for computing crop water requirements. Rome: FAO. 1998. 300p. Irrigation and Drainage Paper, 56

Berlato, M.A; Mollon, L.C.B. Evaporação e evapotranspiração. Porto Alegre: IPAGRO, 1981.95p.

Brutsaert, W. Evaporation into the atmosphere, theory, history, and applications. Dordrecht: D. Reidel, 1982. 299p.

Camargo, A. P.; Sentelhas, P.C. Avaliação do desempenho de diferentes métodos de estimativa da evapotranspiração potencial no estado de São Paulo, Brasil. Revista Brasileira de Agrometeorologia, Santa Maria, v.5, n.1, p.89-97, 1997.

Carter, C.E.; Irvine, J.E; McDaniel, V.; Dunckelman, J. Yield response of sugarcane to stalk density and subsurface drainage treatments. Transactions of the ASAE, St. Joseph, v.28, n.1, p.172-177, 1985.

Doorenbos, J.; Pruitt, W.O. Necessidades hídricas das culturas. Campina Grande: UFPB, 1997. 202p. Estudos FAO. Irrigação e Drenagem, 24.

Gangopadhyaya, M.; Harbeck Jr., G.E.; Nordenson, T.J.; Omar, M. H.; Uryvaev, V. A. Measurement and estimation of evaporation and evapotranspiration. Genebra: WMO, 1966. 121p. Technical Note, 83, WMO, 201, TP 105
Jensen, M.E. (ed.) Comsumptive use of water and irrigation water requirements. New York: American Society of Civil Engineers, 1973.215p.

Jensen, M.E.; Burman, R.D.; Allen, R.G. Evapotranspiration and irrigation water requirements. New York: American Society of Civil Engineers, 1990. 332p. Manual and Reports on Engineering Practice, 70

Medeiros, S.L.P. Avaliação de métodos de estimativa da evapotranspiração de referência para a região mesoclimática de Santa Maria-RS. Revista Brasileira de Agrometeorologia, Santa Maria, v.6, n.1, p.105-109, 1998.

Miranda, J.H. Modelo para simulação da dinâmica da água em sistemas de drenagem subterrânea e cálculo do espaçamento econômico entre drenos. Piracicaba: ESALQ, 1997. 89p. Dissertação Mestrado

Penman, H.L.; Angus, D.E.; van Bavel, C.H.N. Microclimatic factors affecting evaporation and transpiration. In: Hagan, R.M.; Haise, H.H.; Edminster, T.W. (ed.) Irrigation of Agricultural Lands. Madison: American Society Agronomy, 1967. p.483-505.

Pereira, A.R.; Villa Nova, N.A.; Sediyama, G.C. Evapo(transpi)ração. Piracicaba: FEALQ, 1997, 183 p.

Santiago, A. V. Evapotranspiração de referência medida por lisímetro de pesagem e estimada por Penman-Monteith (FAO-56), nas escalas mensal e decendial. Piracicaba: ESALQ, 2001.37p. Dissertação Mestrado

Scardua, R. O clima e a irrigação na produção agroindustrial da cana-de-açúcar (Saccharum spp). Piracicaba: ESALQ, 1985. 122p. Tese Livre-Docência

Villa Nova, N.A; Reichardt, K. Evaporação/evapotranspiração de um ecossistema e suas relações com o meio ambiente. Engenharia Hidrológica, Rio de Janeiro, v.2, p.145-197,1989. 